\title{
Fiber-Optic Current Sensor Head Based on the Giant Magnetostrictive Materials
}

\author{
Naikui Ren ${ }^{1}$, Yanling Xiong ${ }^{1 *}$, Mingze $\mathrm{Wu}^{1}$, Yuelan $\mathrm{Lv}^{2}$ and Lianjin Hong ${ }^{2}$ \\ 1. Harbin Engineering University \\ 2. College of Applied Science, Harbin University of Science and Technology \\ Harbin, 150080, CHINA \\ *Email: xyling1964@163.com
}

\begin{abstract}
A current sensing method based on giant magnetostrictive material (GMM) and fiber Bragg grating $(F B G)$ was proposed, which not only combines the advantages of $F B G$ such as wavelength encoding, compatibility with fiber and distributed measurement system realization, but also the advantages of GMM as large flexible coefficient, high electromechanical coupling coefficient and short response time. The minimal residual method was put forward to effectively identify the Jiles-Atherton model parameters of ferro-magnetic material. The parameters of the giant magnetostrictive (Terfenol-D bar ) and the Jiles-Atherton model was established and optimized by using the Mathematica software. Then the optical fiber current test system with ferromagnetic loop was set up to investigate the current sensing properties of the sensor. The relationship between current excitation and GMM-FBG reflected wavelength was obtained, which provides basic support for further current sensing experiment research.
\end{abstract}

Keywords: Giant magnetostrictive material; Fiber Bragg grating; Current sensor; Minimal residual method

\section{Introduction}

The current sensor plays an important role in the measurement and relay protection with the development of the market-oriented process in power industry, which is demanded increasingly by the sensing technology. Traditional electromagnetic transformer was replaced by optical current sensor with the advantages of good insulating property, electromagnetic interference immunity and wide frequency band. However, the fiber linear birefringence problem, which generates in Faraday magneto-optical effect current sensor, has not been solved well [1]. A new current sensor may be developed successfully to solve these problems based on the advantages of fiber Bragg grating (FBG) with wavelength encoding, good compatibility with fiber and distributed measurement.

The giant magnetostrictive material (GMM) developed in the 1980s, which is a kind of rare earth - iron compound with the magnetostrictive coefficient about 100 to 1000 times higher than the general magnetostrictive material. And GMM is a kind of new magnetomechanical converting material that has been broadly applied in the research field of energy exchanging and driving due to its advantages of high power density, rapid mechanical response, high electromechanical coupling coefficient and short response time [2]. A new current sensor based on GMM and FBG was proposed in this article, which will solve the fiber linear birefringence problem fundamentally.

Reports about applying GMM-FBG system to achieve power frequency alternating current sensing on the international was set up from 2004, and majority studies were focused on the temperature characteristic [3]. Most researches about GMM were 
concentrated in the field of actuator. Researches about the sensing performance of GMM and the further studies of hysteresis characteristics were few [4,5].

Jiles-Atherton model was the equation of magnetization and external magnetic field which was obtained by Physicists D C Jiles and D L Atherton considering domain wall motion and the balance theory of energy [6]. The model results showed a good consistency with experiments because of its simple model with only one ordinary differential equation and the undetermined parameters are less. Therefore, the parameters of ferromagnetic materials of Jiles-Atherton model were identified through minimum residual method in this paper and were optimized by Mathematica software. Finally, the GMM-FBG current sensing system was set up and the performance of GMM-FBG were investigated.

\section{The Hysteresis Nonlinear Sensing Model of GMM}

Nonlinear hysteresis phenomenon between the output displacement and excitation magnetic field intensity exists obviously because of the external magnetic field of GMM, which leads to the output equation is a multi-valued output function. Besides, it has a negative influence on output error and stability of the system. What's more, the design and analysis based on the GMM sensor will be complicated. Thus, a nonlinear hysteresis dynamic sensing model of magnetic material was established through the secondary domain model and the Jiles-Atherton average energy field theory, which can provide the theory support for GMM sensor.

The differential equation between magnetization intensity and the external magnetic field were established by the Jiles-Atherton model was shown as following.

The effective magnetic field strength of ferromagnetic material can be approximately wrote as

$$
\begin{gathered}
H_{e f f}=H+\tilde{\alpha} M \\
\tilde{\alpha}=\alpha+\frac{9}{2} \frac{\gamma_{s} \sigma}{\mu_{0} M_{s}^{2}}
\end{gathered}
$$

Where, $H_{\text {eff }}$ represents the effective magnetic field strength, $H$ is the magnetic field strength excited by external. $\alpha$ is the parameter determined by the interaction of the magnetic domains. $M_{s}$ represents the saturation magnetization of the material.

Magnetization without hysteresis can be calculated with the effective magnetic field strength.

$$
M_{a n}=M_{s} L\left(\frac{H_{e f f}}{a}\right)=M_{s}\left[\operatorname{coth}\left(\frac{H_{e f f}}{a}\right)-\frac{a}{H_{e f f}}\right]
$$

Where, $M_{a n}$ is magnetization without hysteresis.

The relationship among total magnetization, irreversible magnetization and reversible magnetization is as follow

$$
M=M_{r e v}+M_{i r r}
$$

Where, $M_{\text {irr }}$ represents irreversible magnetization, $M_{\text {rev }}$ represents the reversible magnetization, $M$ represents the total magnetization.

irreversible magnetization and reversible magnetization can be calculated as follow equation

$$
\begin{gathered}
\frac{\mathrm{d} M_{i r r}}{\mathrm{~d} H}=\frac{M_{a n}-M_{i r r}}{\delta k-\tilde{\alpha}\left(M_{a n}-M_{i r r}\right)} \\
M_{r e v}=c\left(M_{a n}-M_{i r r}\right)
\end{gathered}
$$


The total magnetization could be achieved through the equation from Eq.1 to Eq.6.

$$
\frac{d M}{d H}=\left(\frac{M_{a n}-M}{\delta K-\alpha \frac{\left(M_{a n}-M\right)}{1-c}}+c \frac{d M_{a n}}{d H}\right) /\left(1-c \frac{d M_{a n}}{d H}\right)
$$

$M_{s}, a, \alpha, k$ and $c$ are saturation magnetostriction constant, saturation magnetization, shape factor without hysteresis magnetization, domain wall interaction coefficient, hysteresis loss parameters and reversible coefficient, respectively. The $H-M$ curve of Jiles-Atherton model can be achieved through the Eq.7, shown in Figure 1.

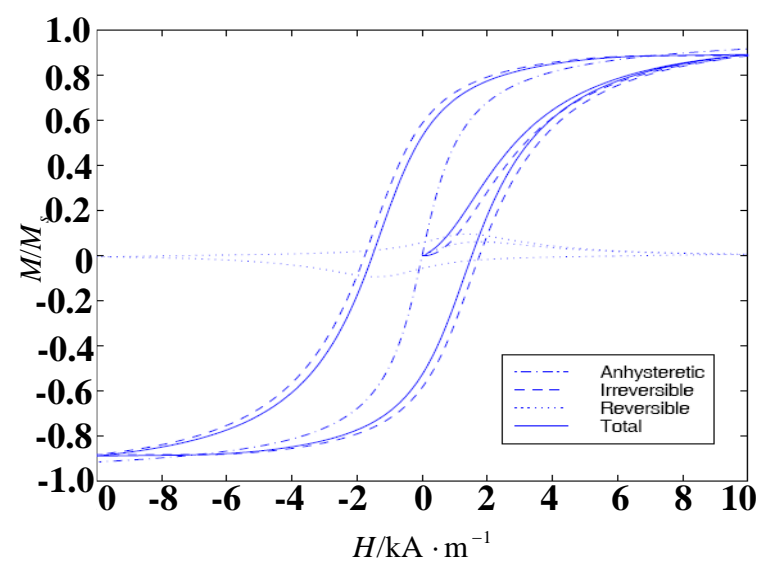

Figure 1. H-M Curve of Jiles-Atherton Model

$$
\gamma=3 \gamma_{s} M^{2} / 2 M_{s}^{2}
$$

Equation (8) is the secondary domain model. $\gamma \mathrm{s}, \mathrm{Ms}, \mathrm{a}, \alpha, \mathrm{k}$ and $\mathrm{c}$ are saturation magnetostriction constant, saturation magnetization, shape factor without hysteresis magnetization, domain wall interaction coefficient, hysteresis loss parameters and reversible coefficient, respectively. They are all undetermined parameters except $\gamma$ s and Ms which can be obtained by experiments. A minimum residual method for determining the undetermined parameters is proposed in this paper. $\mathrm{Hi}, \mathrm{Mi}$ ', Mi, (a, $\alpha, \mathrm{c}, \mathrm{k})$ are defined as the external magnetic field intensity, experiments and model magnetic field about the ith point, respectively. And the formula of $\mathrm{f}(\mathrm{a}, \alpha, \mathrm{c}, \mathrm{k})$ is shown as following,

$$
f(\alpha, a, c, k)=\sum_{i=1}^{N}\left(M_{i}^{\prime}-M_{i}(\alpha, a, c, k)\right)^{2}
$$

Thus, the satisfactory value of a, $\alpha, \mathrm{c}, \mathrm{k}$ can be achieved through solving the minimum value of the equations.

The Tb0.27Dy0.73Fe2 (Terfenol-D) fabricated by Beijing university of Science and Technology was selected as GMM. The hysteresis characteristic curve of Terfenol-D under 0 pre-stressed was obtained by magnetostrictive measurement instrument (JDM28). The saturation magnetization intensity and saturation magnetostriction coefficient of Terfenol-D were $1.17 \times 108 \mathrm{~A} / \mathrm{m}$ and $1.005 \times 10^{-6}$, respectively, which were achieved by the experiment data. The Jiles-Atherton model was achieved through Mathematica by using the minimum residual method to extract data from the hysteresis characteristic curve is shown in Figure 2 


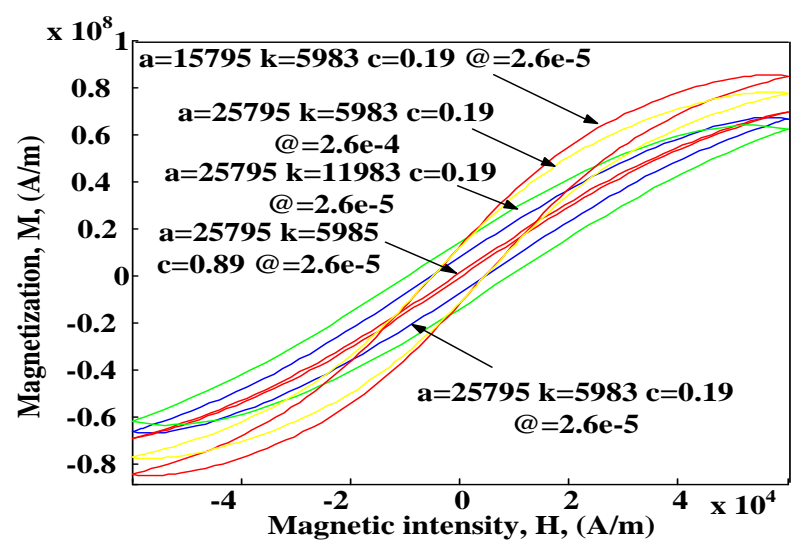

Figure 2. The Influence of the Different Parameters in the Jiles-Atherton Model

It can be seen from the Figure 2, the annularity of the hysteresis characteristic curve was narrower and the slope increased with the increasing of the parameter c. Meanwhile, the influence susceptibility from the external magnetic field to the magnetization intensity of material became stronger. The annularity of hysteresis characteristic curves wider more significantly with the increasing of $\mathrm{k}$. When the a raised, the annularity of hysteresis characteristic curves change little but the slope decreased obviously. And the slope increased with the increasing of $\alpha$. These parameters that $\alpha=2.6 \times 10^{-5}, \mathrm{c}=0.19, \mathrm{k}=5983 \mathrm{~A} / \mathrm{m}$ and $a=25795$ can be obtained through optimizing, and it can be achieved that the simulation curve of Jiles-Atherton model is consistent with the H-M curve of experiment, which shown in Figure 3.

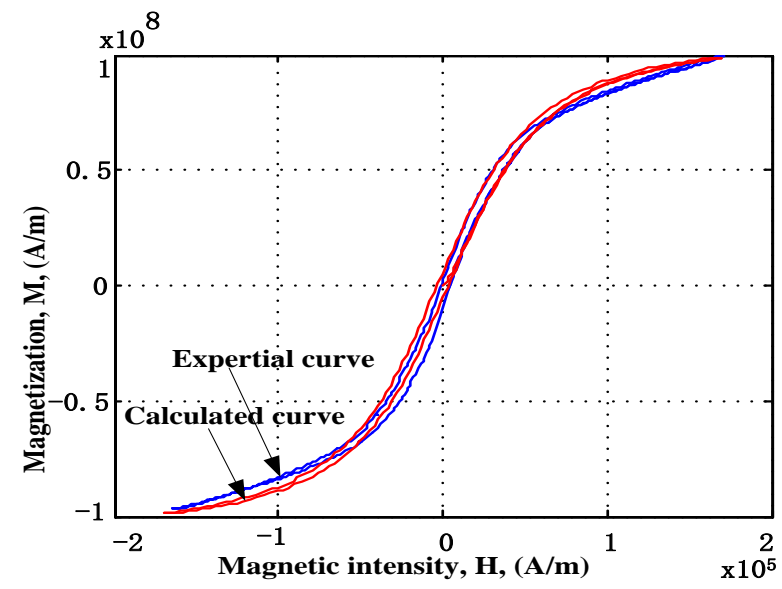

Figure 3. The Simulated and Experimental Curves

The relationship between magnetic field intensity and magnetostriction coefficient was established based on the secondary domain model, which is shown in Figure 4. 


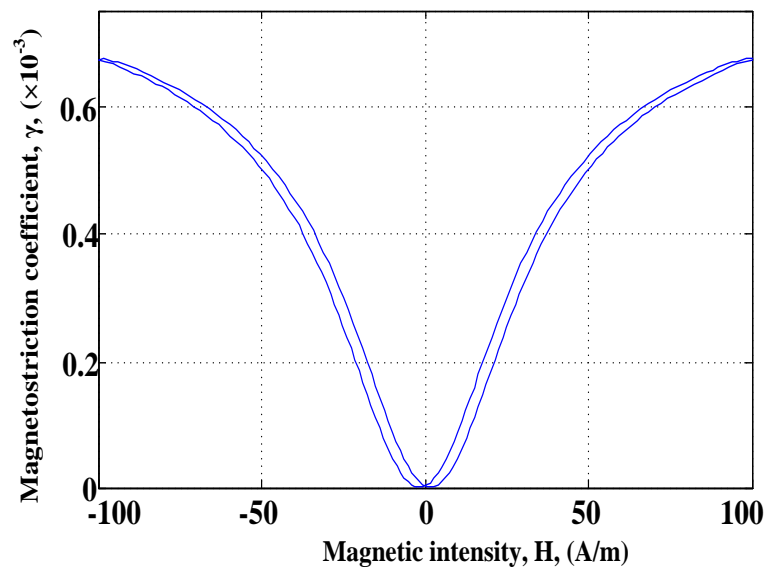

Figure 4. Relationship between Magnetostriction Coefficient $y$ and Magnetic Field Intensity H

\section{Sensing Experiment of GMM-FBG}

\subsection{Design of GMM-FBG Sensing}

When the temperature is constant, the relationship between the axial strain $\varepsilon$ and FBG wavelength variation $\Delta \lambda \mathrm{B}$ is,

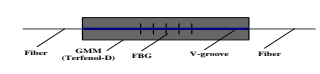

Where, $\mathrm{P}_{\mathrm{e}}$ is fiber photoelastic coefficient, $\lambda_{\mathrm{B}}$ is the center wavelength of FBG. The equation shows that $\lambda_{B}$ changes with axial strain.

GMM and FBG were combined as current sensor, based on the high magnetostrictive effect of GMM and the good sensing characteristic of FBG. The sensor with the advantages of easy operation and compact size, which can be placed in a magnetic field to monitor current or magnetic. The design scheme of sensor is shown in Figure 5. Specific fabrication method of sensor was shown as follows. Firstly, a V-shaped groove was carved along the axis on the side of GMM. Secondly, the sensing grating was straightened in the groove. Finally, FBG was stuck on the GMM rod. In the design process of sensor, the wavelength range of demodulation system and the wavelength shift in the process of straightening FBG should be considered for the selection of FBG center wavelength. The FBG reflectance should be above $90 \%$, the range of $3 \mathrm{~dB}$ bandwidth is from $0.15 \mathrm{~nm}$ to $0.2 \mathrm{~nm}$ and the grid region is $1 \mathrm{~cm}$. The length of Terfenol-D bar, which was chosen as $\mathrm{GMM}$, is $2 \mathrm{~cm}$.

If the GMM-FBG sensor system was exposed to a magnetic field, the pulsating strain of GMM caused by magnetic field was converted into the wavelength variation of FBG, which can be achieved by FBG wavelength interrogation system. And the relationship between GMM-FBG reflection wavelength and excitation current or magnetic field intensity can be established.

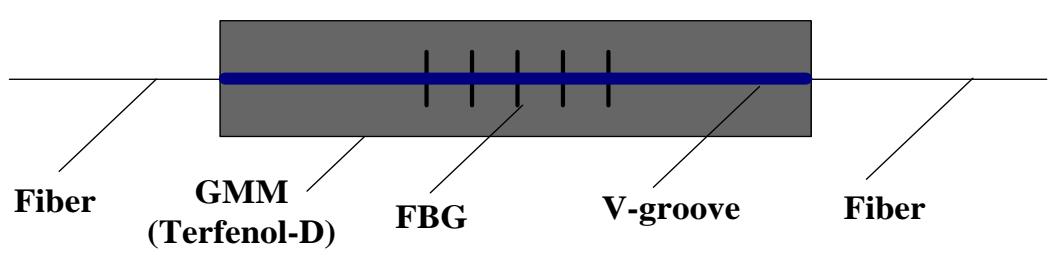

Figure 5. The Scheme of GMM-FBG Sensor 


\subsection{GMM-FBG Sensing System}

The experimental devices of GMM-FBG sensing current sensing can be divided into electromagnetic systems, light source, sensing system and demodulation system, which was shown in Figure 6. The lights were emitted by ASE reached to GMM-FBG sensor through the coupler and then reflected by FBG. The reflected lights were achieved by FBG wavelength analyzer which can demodulate the wavelength information. During the experiment, the magnetic field was generated by current carrying conductor twined on silicon steel lamination stack and the turn number of conductor coil is 850 , and ampereturns current was used [7].

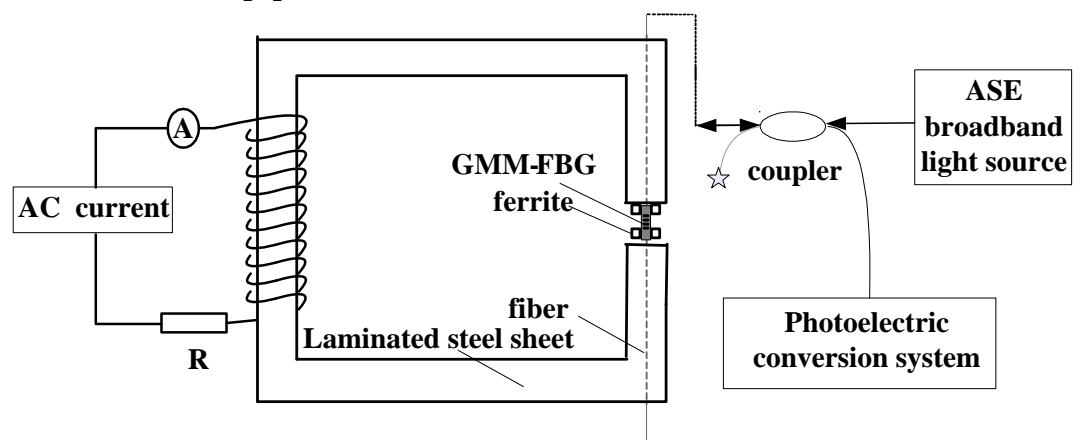

Figure 6. Experimental System of the Sensor

The relationship between the magmatic field intensity of GMM-FBG sensing element $\mathrm{H}$ and the ampere-turns current $\mathrm{I}$ was $\mathrm{H}=0.06 \mathrm{I}$, which was measured before the experiments. The hysteresis loop of GMM was shown in Figure 7 when the I was increased from 0 to $3400 \mathrm{~A}$. It can be seen that the abscissa represents ampere-turns current though wire and the corresponding magnetic field. While the ordinate represents the wavelength of FBG and the strain $\varepsilon$ after conversion.

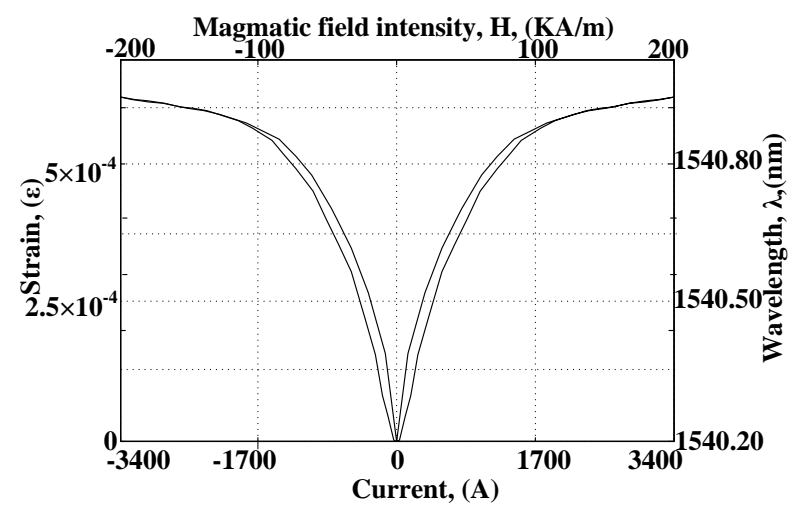

Figure 7. Hysteresis Loop of the GMM

As shown in Figure 7, the strain $\varepsilon$ changed slowly with magmatic field intensity $\mathrm{H}$ under the condition of 0 pre-stress, when $\mathrm{H}$ in a small range (less than $10 \mathrm{kA} / \mathrm{m}$ ). With the continuous increasing of $\mathrm{H}$, the variation of $\varepsilon$ is large, and the relationship between $\mathrm{H}$ and $\varepsilon$ is almost linear. But the strain went into mild change region until the saturation is achieved, when the magnetic field intensity is more than $80 \mathrm{kA} / \mathrm{m}$. It could been obtained that the saturated magnetic field strength is about $200 \mathrm{kA} / \mathrm{m}$, the saturated strain is about $610 \mu \varepsilon$ and the conclusions are consistent with the simulation results which are shown in Figure 4.

The experiment system was set up according to the diagram shown in Figure 6. The wavelengths of GMM-FBG reflected lights were achieved through wavelength demodulation instrument, when the input direct currents were changed from $0 \mathrm{~A}$ to $1445 \mathrm{~A}$. 
The relationship between input current amplitude and output signal amplitude was established after multi-measurement shown in Figure 8. And the relationship is linear, what's more, a favorable consistency was gained between the curves. And then took the average of excitation current over $340 \mathrm{~A}$, these data were used to calculate the average output signal amplitude. Finally the relationship between input current and average output amplitude was established shown in Figure 9, and then a sensitivity of $0.488 \mathrm{pm} / \mathrm{A}$ was obtained.

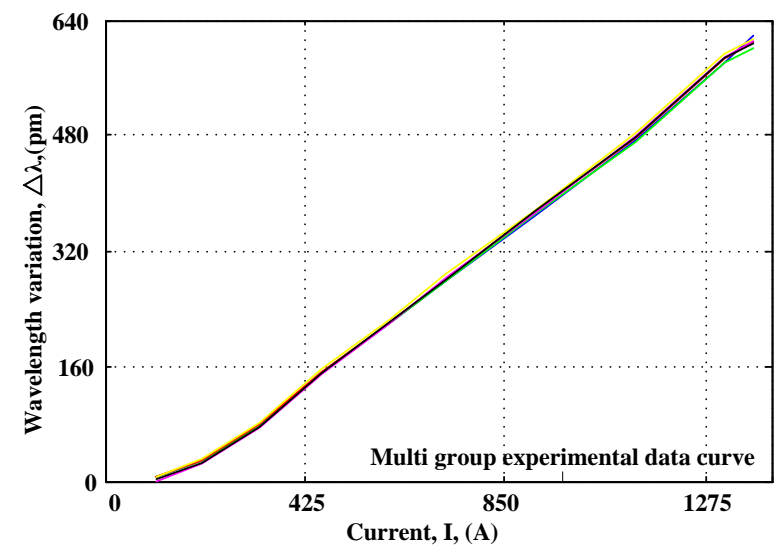

Figure 8. The Relationship between Input Current Amplitude and Output Signal Amplitude

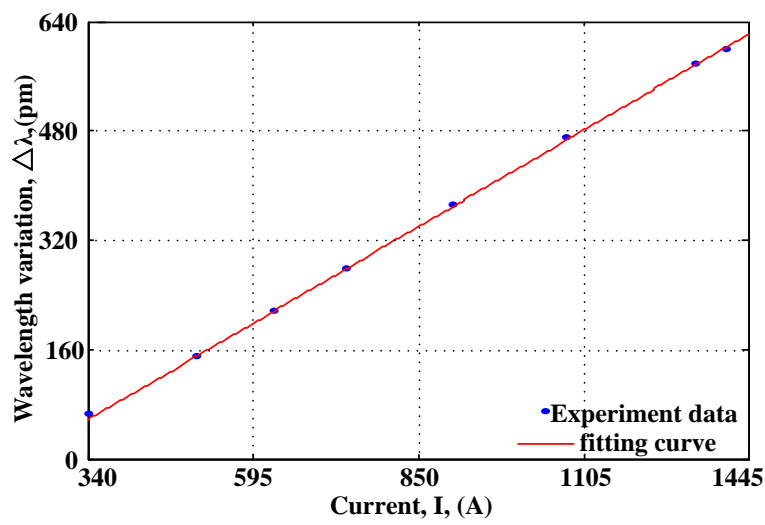

Figure 9. The Relationship between Input Current and Average Output Amplitude

\section{Conclusion}

In this paper, the identification and optimization of the parameters of Terfenol-D was based on the Jiles-Atherton model through the minimum residual method and then obtained the hysteresis rule was consistent with the experimental results. A current sensing system with ferromagnetic loops was set up to investigate the sensing characteristic of GMM-FBG. The relationship between excitation current and the reflection wavelength of GMM-FBG was established and a sensitivity of $0.488 \mathrm{pm} / \mathrm{A}$ was achieved.

\section{Acknowledgments}

Thanks Heilongjiang Provincial Department of Education funding for research projects (12541163). 


\section{References}

[1] T. Y. Wang, "The study of high voltage photoelectric current transformer", Beijing: Qinghua university postdoctoral research report, (2000), pp. 3-14.

[2] S. Y. Cao, B. W. Wang and R. G. Yan, Proceedings of the CSEE, China, vol. 11, no. 23, (2003).

[3] D. Reilly, A. J. Willshire, G. Fusiek, P. Niewczas and J. R. Mcdonald, Sensors Journal, IEEE, vol. 6, no. 6, (2006).

[4] P. Niewczas, G. Fusiek and J. R. McDonald, IEEE SENSORS 2006, the 5th IEEE Conference on Sensors, Daegu, Korea, (2006).

[5] G. Fusiek, P. Niewczas and J. R. McDonald, Instrumentation and Measurement Technology Conference Proceedings, Warsaw, Poland, (2007).

[6] D. C. Jiles and D. L. Atherton, Journal of Magnetism and Magnetics, vol. 61, (1986), pp. 1-2.

[7] Y. L. Xiong, “An Terfenol-D optical Fiber Bragg Grating based curre sensing”, Univ. of HUST, (2006), pp. 66-67. 